\title{
CHARACTERIZATION OF THE COLLAPSING MEROMORPHIC PRODUCTS
}

\author{
Alain Escassut and Marie-Claude Sarmant
}

Abstract

Let $K$ be an algebraically closed complete ultrametric field. Let $a \in K, r>0$. We consider a meromorphic product $F(x)=$ $\prod_{n \in \mathbb{N}} \frac{x-a_{n}}{x-b_{n}}$, where $\left(a_{n}\right)_{n \in \mathbb{N}},\left(b_{n}\right)_{n \in \mathbb{N}}$ are sequences satisfying $\left|b_{n}-a\right|<r$ whenever $n \in \mathbb{N}, \lim _{n \rightarrow+\infty}\left|b_{n}-a\right|=r, \lim _{n \rightarrow \infty} a_{n}-b_{n}=0$ and $\min _{m \neq n}\left|b_{m}-b_{n}\right|>0$. We prove that if $K$ has characteristic zero, then $F$ is collapsing if and only if $\sum_{n=0}^{\infty}\left(a_{n}\right)^{j}-\left(b_{n}\right)^{j}=0$ for every $j \in \mathbb{N}$. Moreover, if $K$ has characteristic $\neq 0$, then there exists a meromorphic product $f$ of the form $\prod_{n \in \mathbb{N}} \frac{x-c_{n}}{x-e_{n}}$ such that $F(x)=(f(x))^{p}$ whenever $x \in\{x \in K|| x-a \mid \geq r\}$ if and only if $\sum_{n=0}^{\infty}\left(a_{n}\right)^{j}-\left(b_{n}\right)^{j}=0$ for every $j \in \mathbb{N}$.

\section{Notations and definitions}

Let $K$ be an algebraically closed field, complete with respect to an ultrametric absolute value. Given a set $D$ in $K, H(D)$ denotes the set of the analytic elements in $D$, i.e., the completion of the algebra $R(D)$ of rational functions with no pole in $D$, with respect to the topology of uniform convergence.

Given $a \in K$ and $r>0, d(a, r)$ (resp. $d\left(a, r^{-}\right)$) denotes the disk $\{x \in K|| x-a \mid \leq r\}$ (resp. $\{x \in K|| x-a \mid<r\}$ ).

We put $V=d\left(a, r^{-}\right)$and $E=K \backslash V$. A sequence $\left(e_{n}\right)_{n \in \mathbb{N}}$ in $V$ satisfying $\lim _{n \rightarrow \infty}\left|e_{n}-a\right|=r$ and $\min _{m \neq n}\left|e_{m}-e_{n}\right|>0$ will be called a polar sequence associated to $V$. 
Henceforth, $\left(b_{n}\right)_{n \in \mathbb{N}}$ will denote a polar sequence associated to $V$ and $\left(a_{n}\right)_{n \in \mathbb{N}}$ will denote a sequence in $K$ such that $\lim _{n \rightarrow \infty} a_{n}-b_{n}=0$.

For every $x \in K \backslash\left\{b_{0}, \ldots, b_{n}, \ldots\right\}$ the product $F_{m}=\prod_{n=0}^{m} \frac{x-a_{n}}{x-b_{n}}$ converges to a limit $F(x)=\prod_{n \in \mathbb{N}} \frac{x-a_{n}}{x-b_{n}}$. Such a function $F(x)$ defined in $K \backslash\left\{b_{1}, \ldots, b_{n}, \ldots\right\}$ is called a meromorphic product associated to the sequence $\left(b_{n}\right)_{n \in \mathbb{N}}$.

The meromorphic product $\prod_{n \in \mathbb{N}} \frac{x-a_{n}}{x-b_{n}}$ associated to the sequence $\left(b_{n}\right)_{n \in \mathbb{N}}$ will be said to be collapsing if there exists $\ell \in K$ such that $F$ satisfies $\lim _{|x-a| \rightarrow r} F(x)=\ell$.

By $[\mathbf{5}],[\mathbf{7}]$ it is well known that a meromorphic product $f$ is collapsing if and only if $f-1$ is vanishing along the increasing filter $\mathcal{F}$ of center 0 and diameter 1 , and in particular this requires $\mathcal{F}$ to be a $T$-filter [4]. Now, the question whether a meromorphic product is collapsing, in connection with the sequences $\left(a_{n}\right)_{n \in \mathbb{N}},\left(b_{n}\right)_{n \in \mathbb{N}}$, is a quite hard question. Here we will give an answer. In particular, this will be used in the study of the homomorphisms from the group of meromorphic products into the circle $C(0,1)$.

By $[\mathbf{5}],[\mathbf{7}]$ we have Lemma a.

Lemma a. The following are equivalent.

(1) $F$ is collapsing,

(2) $\lim _{|x-a| \rightarrow r} F(x)=1$,

(3) $F(x)=1$ whenever $x \in E$.

Next result is taken from [5].

Theorem 0. Let $f \in H(E)$ satisfy $\lim _{|x| \rightarrow \infty} f(x)=1$ and $\|f-1\|_{E}<1$. Let $\epsilon \in] 0,\|f-1\|_{E}\left[\right.$. There exist a polar sequence $\left(e_{n}\right)_{n \in \mathbb{N}}$ associated to $V$, together with a meromorphic product $\prod_{n=0}^{\infty} \frac{x-c_{n}}{x-e_{n}}$ associated to the sequence $\left(e_{n}\right)_{n \in \mathbb{N}}$, satisfying further $\left|c_{n}-e_{n}\right|<r\left(\|f-1\|_{E}+\epsilon\right)$, and $\prod_{n=0}^{\infty} \frac{x-c_{n}}{x-e_{n}}=f(x)$ whenever $x \in E$. 
We notice that for every $j \in \mathbb{N}^{*}$ the series $\sum_{n=0}^{\infty} a_{n}^{j}-b_{n}^{j}$ is convergent. Lemma b below is easy and will be used in proving Lemma c.

Lemma b. Let $\lambda \in K$. The following are equivalent.

i) $\sum_{n=0}^{\infty} a_{n}^{j}-b_{n}^{j}=0$ for every $j \in \mathbb{N}^{*}$

ii) $\sum_{n=0}^{\infty}\left(a_{n}+\lambda\right)^{j}-\left(b_{n}+\lambda\right)^{j}=0$ for every $j \in \mathbb{N}^{*}$.

Lemma c. $F$ satisfies $F^{\prime}(x)=0$ for all $x \in E$ if and only if for every $j \in \mathbb{N}^{*}$ the sequences $\left(a_{n}\right)_{n \in \mathbb{N}}$ and $\left(b_{n}\right)_{n \in \mathbb{N}}$ satisfy $\sum_{n=0}^{\infty}\left(a_{n}\right)^{j}-\left(b_{n}\right)^{j}=0$.

Proof: By Lemma b we may clearly assume $a=0$ without loss of generality. Let $r^{\prime} \in\left[r,+\infty\right.$ [ be such that $\left|a_{n}\right|<r^{\prime}$ for every $n \in \mathbb{N}$, and let $E^{\prime}=K \backslash d\left(0, r^{\prime-}\right)$. We can see that $\|F-1\|_{E}^{\prime} \leq \sup _{n \in \mathbb{N}} \frac{\left|b_{n}-a_{n}\right|}{r^{\prime}}<1$. Hence $\frac{F^{\prime}}{F}$ obviously belongs to $H\left(E^{\prime}\right)$. Let $g=\frac{F^{\prime}}{F}$. It is seen that $g(x)=\sum_{n=0}^{\infty} \frac{1}{x-a_{n}}-\frac{1}{x-b_{n}}$. For each $\alpha, \beta \in V$, and for every $x \in E^{\prime}$ we have

$$
\frac{1}{x-\alpha}-\frac{1}{x-\beta}=\sum_{j=0}^{\infty} \frac{\alpha^{j}-\beta^{j}}{x^{j+1}}=\sum_{j=1}^{\infty} \frac{\alpha^{j}-\beta^{j}}{x^{j+1}}
$$

Applying this to each term $\frac{1}{x-a_{n}}-\frac{1}{x-b_{n}}$, we obtain

$$
g(x)=\sum_{n=0}^{\infty}\left(\sum_{j=1}^{\infty} \frac{\left(a_{n}\right)^{j}-\left(b_{n}\right)^{j}}{x^{j+1}}\right)
$$

for all $x \in E^{\prime}$. Now, let us fix $x \in E^{\prime}$. We see that when $j$ tends to $+\infty$, the convergence of $\frac{\left(a_{n}\right)^{j}-\left(b_{n}\right)^{j}}{x^{j+1}}$ to 0 is uniform with respect to $n$. Hence we have

$$
g(x)=\sum_{j=1}^{\infty}\left[\sum_{n=0}^{\infty} \frac{\left(a_{n}\right)^{j}-\left(b_{n}\right)^{j}}{x^{j+1}}\right]
$$


But now, this holds for any $x \in E^{\prime}$. Besides, as $F$ belongs to $H(E)$, we know that its Mittag-Leffler series [3], [4] is the same in $H(E)$ and in $H\left(E^{\prime}\right)$, hence this is the Mittag-Leffler series of $F$ in $H(E)$. Hence we see that $F^{\prime}(x)=0$ if and only if the Mittag-Leffler series of $g$ is identically equal to 0, i.e.: $\sum_{n=0}^{\infty}\left(a_{n}\right)^{j}-\left(b_{n}\right)^{j}=0$ for every $j \in \mathbb{N}^{*}$. This ends the proof.

Now, we can conclude

Theorem 1. $K$ is supposed to have characteristic zero. Then $F$ is collapsing if and only if for every $j \in \mathbb{N}^{*}$ the sequences $\left(a_{n}\right)_{n \in \mathbb{N}}$ and $\left(b_{n}\right)_{n \in \mathbb{N}}$ satisfy $\left(\mathcal{E}_{j}\right) \sum_{n=0}^{\infty}\left(a_{n}\right)^{j}-\left(b_{n}\right)^{j}=0$.

Proof: Indeed, since $K$ has characteristic zero, by [1] we know that $F^{\prime}(x)$ is identically zero in $E$ if and only if $F(x)$ is a constant in $E$, i.e., $F$ is collapsing.

Theorem 2. Assume $K$ to be of characteristic $p \neq 0$. There exists a polar sequence $\left(e_{n}\right)_{n \in \mathbb{N}}$ associated to $V$, and a meromorphic product $f(x)=\prod_{n \in \mathbb{N}} \frac{x-c_{n}}{x-e_{n}}$, associated to the sequence $\left(e_{n}\right)_{n \in \mathbb{N}}$, satisfying $F(x)=$ $(f(x))^{p}$ whenever $x \in E$ if and only if for every $j \in \mathbb{N}^{*}$ the sequences $\left(a_{n}\right)_{n \in \mathbb{N}}$ and $\left(b_{n}\right)_{n \in \mathbb{N}}$ satisfy $\left(\mathcal{E}_{j}\right) \sum_{n=0}^{\infty}\left(a_{n}\right)^{j}-\left(b_{n}\right)^{j}=0$.

Proof: If there exists a meromorphic product $f$ associated to the sequence $\left(b_{n}\right)_{n \in \mathbb{N}}$ such that $(f(x))^{p}=F(x)$ for all $x \in E$, then obviously we have $F^{\prime}(x)=0$ for all $x \in E$, and therefore, by Lemma c, we have $\left(\mathcal{E}_{j}\right) \sum_{n=0}^{\infty}\left(a_{n}\right)^{j}-\left(b_{n}\right)^{j}=0$ for every $j \in \mathbb{N}^{*}$.

Reciprocally, we suppose Relations $\left(\mathcal{E}_{j}\right)$ satisfied. By Lemma b we have $F^{\prime}(x)=0$ for all $x \in E$. Hence, there exists $g \in H(E)$ such that $(g(x))^{p}=F(x)$ for all $x \in E$. Besides, since $F$ is a meromorphic product associated to the sequence $\left(b_{n}\right)_{n \in \mathbb{N}}$, we notice that $\lim _{|x| \rightarrow+\infty} F(x)=1$. As a consequence, we can choose $g$ such that $\lim _{|x| \rightarrow+\infty} g(x)=1$. Further, it is seen that $g^{p}=((g-1)+1)^{p}=(g-1)^{p}+1$, and therefore we have 


$$
\|F-1\|_{E}=\left(\|g-1\|_{E}\right)^{p},
$$

hence $\|g-1\|_{E}<1$. Let $\left.\epsilon \in\right] 0,1[$. Then by (1) and by Theorem 0 there does exists a polar sequence $\left(e_{n}\right)_{n \in \mathbb{N}}$ associated to $V$, and a meromorphic product $f$ of the form $\prod_{n \in \mathbb{N}} \frac{x-c_{n}}{x-e_{n}}$ such that $f(x)=g(x)$ whenever $x \in E$, and such that $\left|e_{n}-c_{n}\right| \leq \sqrt[p]{\|F-1\|_{E}}+\epsilon$. This ends the proof.

Remark. In [5], and [6] it was shown how one can construct a collapsing meromorphic product, with the help of certain unbounded functions analytic in the disk $d\left(0, r^{-}\right)$.

Acknowledgement. We are very grateful to Labib Haddad for his contribution to this article.

\section{References}

1. A. Escassut, Derivative of analytic elements on infraconnected clopen sets, Indag. Math. 51 (1989), 63-70.

2. A. Escassut, $T$-filtres, ensembles analytiques et transformation de Fourier p-adique, Ann. Inst. Fourier 25(2) (1975), 45-80.

3. A. Escassut and M.-C. Sarmant, Mittag-Leffler series and Motzkin products for invertible analytic elements, Rivista di Matematica Pura ed Applicata 12 (1992), 61-73.

4. M. Krasner, Prolongement analytique uniforme et multiforme dans les corps valués complets. Les tendances géométriques en algèbre et théorie des nombres, Clermont-Ferrand (1964), 94-141; Centre National de la Recherche Scientifique (Colloques internationaux de C.N.R.S. Paris, 143) (1966).

5. M.-C. Sarmant, Produits Méromorphes, Bull. Sci. Math. 109 (1985), 155-178.

6. M.-C. Sarmant, Fonctions analytiques et produits croulants, Collectanea Mathematica XXXVI(2) (1985), 199-218.

7. M.-C. SARMant, Produits meromorphes et prolongement analytique, Thèse de Doctorat d'Etat, Université Pierre et Marie Curie (1987). 
8. M.-C. SARmant, Factorisation en produit méromorphe d'un elémént semi-inversible, Bull. Sci. Math. 115 (1991), 379-394.

\author{
Université Blaise Pascal (Clermont-Ferrand) \\ Laboratoire de Mathématiques Pures \\ Les Cézeaux \\ 63177 Aubière Cedex \\ FRANCE
}

Primera versió rebuda el 13 de Setembre de 1995, darrera versió rebuda el 12 de Gener de 1996 